\title{
La gestión del cambio, la colaboración virtual y la agilidad estratégica organizacional de empresas mexicanas ante los impactos por el COVID-19 Change management, virtual collaboration, and strategic organizational agility of Mexican companies in the face of COVID-19 impacts
}

\author{
Eréndira Fierro-Moreno ${ }^{1}$ [1] https://orcid.org/0000-0002-4397-1179 \\ ${ }^{1}$ Universidad Autónoma del Estado de México, Toluca: efierrom@uamex.mx \\ (c) Universidad De La Salle Bajío (México)
}

Palabras clave: cambio; estrategias; virtual; digital; COVID-19; empresas; agilidad estratégica; gestión empresarial; colaboración; estudios organizacionales; crisis global; teoría de la contingencia

Keywords: change; strategies; virtual; digital; COVID-19; companies; strategic agility; business management; collaboration; organizational studies; global crisis; contingency theory

Recibido en: 13 - 11 - 2020 / Aceptado en: $14-03$ - 2021

\section{Resumen}

Introducción: ¿Cómo enfrentar una crisis mundial médica? ¿Cómo enfrentarla, si además se generó también una crisis económica mundial nunca antes vista? ¿Gestionar el cambio implica gestionar lo absurdo? Ante la pandemia, las organizaciones debieron hacer frente a la tensión y considerar que la estabilidad debe asumir los procesos de cambio, al ser éste inevitable y formar parte de la vida de la organización. La continuidad y el cambio no deben gestionarse como estados alternativos sino como estados coexistentes. Fue fundamental llevar a cabo estrategias tales como la colaboración virtual, la transformación digital y la agilidad estratégica organizacional.

Método: El objetivo de este artículo consistió en determinar en qué medida la gestión del cambio influye en la colaboración virtual, en la transformación digital y en la agilidad estratégica organizacional ante los impactos por la crisis por COVID-19. Con la revisión de literatura y apoyados en la Teoría de la Contingencia se formularon las hipótesis. La fuente de información fue mediante una encuesta de muestreo no probabilístico a 109 empresas de México y la unidad de análisis fue en su mayoría mandos directivos y medios. La metodología utilizada fue un modelo de ecuaciones estructurales (SEM).

Resultados: Los resultados indican que las empresas mexicanas emplearon estrategias asociadas con la gestión del cambio como: el teletrabajo en línea, la optimización del negocio, la inversión en tecnología y la diversificación como respuesta a la crisis derivada del COVID-19. Aunque no es posible establecer una relación de causalidad entre las variables en estudio, ya que la 
interpretación de la causalidad en el mundo real no se garantiza, sí fue posible establecer con estos resultados una discusión acerca de la influencia de la gestión del cambio en la colaboración virtual, en la transformación digital y en la agilidad estratégica organizacional ante la crisis por la COVID19.

Discusión o conclusión: Ante este entorno emergente las empresas replantearon sus estrategias para enfrentarse a la crisis generada por los impactos de la COVID-19. La capacidad de adaptarse rápidamente a los cambios y de gestionarlo de la mejor manera es una capacidad importante y fundamental para mantener y a la vez, transformar a las organizaciones; al ser los cambios rápidos e intensos, es necesario que se gestione el cambio para que las organizaciones sean competitivas a corto plazo y sobrevivan a largo plazo. Los hallazgos de la investigación comprueban e indican que la gestión del cambio predice la colaboración virtual, la transformación digital y la agilidad estratégica organizacional; el análisis empírico de esta investigación ha puesto de manifiesto la importancia de ésta para generar capacidades necesarias que las organizaciones requieren ante respuestas prontas y necesarias para garantizar su supervivencia y competitividad.

\begin{abstract}
Introduction: How to face a world medical crisis, if it also generated a world economic crisis never seen before? Does managing change imply managing the absurd? Faced with the pandemic, organizations had to face the tension and consider that stability must assume the processes of change, it is inevitable and is part of the life of the organization. Continuity and change should not be managed as alternative states but as coexisting states. It was fundamental to carry out strategies such as virtual collaboration, digital transformation and organizational strategic agility.

Method: The objective of this article was to determine to what extent change management influences virtual collaboration, digital transformation and organizational strategic agility in the face of the impacts of the crisis by COVID-19. With the review of literature and supported by the Theory of Contingency, the hypotheses were formulated. The source of information was a nonprobabilistic sample survey of 109 companies in Mexico, and the unit of analysis was mostly management and middle management. The methodology used was a structural equation model (SEM).

Results: The results indicate that Mexican companies employed strategies associated with change management such as: online teleworking, business optimization, investment in technology and
\end{abstract}


diversification as a response to the crisis resulting from COVID-19. Although it is not possible to establish a causal relationship between the variables under study because the interpretation of causality in the real world is not guaranteed, but it was possible to establish with these results a discussion about the influence of change management on virtual collaboration, digital transformation and organizational strategic agility in the face of the crisis by COVID-19.

Discussion or conclusion: Faced with this emerging environment, companies rethought their strategies to face the crisis generated by the impacts of COVID-19. The ability to adapt quickly to change and to manage it in the best way is an important and fundamental capacity to maintain and at the same time, transform organizations. As changes are fast and intense, it is necessary to manage change in order for organizations to be competitive in the short term and to survive in the long term. Research findings prove and indicate that change management predicts virtual collaboration, digital transformation and organizational strategic agility; the empirical analysis of this research has shown the importance of this to generate the necessary capacities that organizations require in the face of prompt and necessary responses to guarantee their survival and competitiveness.

\section{Introducción}

¿Cómo enfrentar una crisis mundial médica? ¿Cómo enfrentarla, si además se generó también una crisis económica mundial nunca antes vista? ¿Gestionar el cambio implica gestionar lo absurdo? La actual pandemia de COVID-19, producida por una cepa mutante de coronavirus el SARS-CoV2, ha generado en todo el mundo, en el siglo 21, una severa crisis económica, social y de salud (Maguiña, Gastelo y Tequen, 2020). El coronavirus es la actual infección que tiene la mayor extensión a nivel mundial y es artífice de cuantiosos cambios en el aspecto socio-político y económico (Mejía et al., 2020).

La pandemia mundial ha creado una crisis médica y junto con ella, una profunda crisis económica. El virus ha afectado a 216 países y se ha requerido hacer frente a las catastróficas consecuencias socioeconómicas, humanitarias y de derechos humanos, con la atención centrada en salvar vidas, a asegurar la accesibilidad a los servicios vitales, a mantener los hogares a flote, a garantizar la solvencia de las empresas y el funcionamiento de las cadenas de suministro, la solidez de las instituciones y de las organizaciones y la prestación de servicios públicos (ONU, 2020) 
En México, se ha advertido que 100 mil negocios podrían cerrar de manera permanente. Se registran pérdidas por 30 mil millones de pesos. Con la estimación de que una vez que se termine la crisis sanitaria se llegará a pérdidas de 250 mil millones de pesos. La incertidumbre, ha provocado que miles de comercios, prestadores de servicios e industrias estén trabajando en $20 \%$ y hasta $40 \%$ de su capacidad instalada y se vean en la necesidad de parar sus actividades y su producción de manera total (ALAMPYME, 2020).

Ante la contingencia, la Organización Panamericana de la Salud (2001) ha definido diversas estrategias, las cuales deben permitir, entre otros, aumentar el conocimiento que tienen los usuarios sobre temas de salud. Seguir principios básicos de salud puede ayudar a mantener a los empleados en sus trabajos para detener la propagación de la enfermedad. Las recomendaciones son, entre otras, la promoción del distanciamiento social (un término aplicado a ciertas acciones que se toman para frenar la propagación de una enfermedad altamente contagiosa, incluyendo la limitación de grandes grupos de personas que se reúnen) (UNICEF, 2020). Derivado del distanciamiento social, es imprescindible el uso de elementos tecnológicos que permitan continuar con las actividades laborales.

Ante la pandemia, las organizaciones debieron hacer frente a la tensión y considerar que la estabilidad debe asumir los procesos de cambio, al ser éste inevitable y formar parte de la vida de la organización. La continuidad y el cambio no deben gestionarse como estados alternativos sino como estados coexistentes, es decir, gestionar el cambio y la continuidad simultáneamente es una tarea mucho más delicada que gestionar el cambio solo, gestionar el cambio implica gestionar lo absurdo (Nasim y Sushil, 2014; Sushil, 2005; Leana y Barry, 2000; Sturdy y Grey, 2003).

Por lo anterior, ha sido necesaria la adopción de un proceso de cambio que incluya la diseminación de información mediante el desarrollo de elementos esenciales que permitan la continuidad laboral a través de entornos virtuales, además de la adopción y transformación digital. No obstante, es fundamental considerar que estos entornos virtuales cada vez más complejos deben, además de dar solución, añadir valor a la consecución de actividades así como a la resolución colaborativa de problemas.

Derivado de estos entornos volátiles e impredecibles, también la agilidad organizacional es necesaria para dar respuestas certeras a los cambios continuos e impredecibles para anticipar o responder a éstos de manera oportuna y con facilidad. 
Es entonces, ante este entorno emergente que las empresas han replanteado sus estrategias para enfrentarse a la crisis generada por los impactos de la COVID-19. Cuando se habla del cambio, es frecuente, que se esté hablando de una adaptación constante al entorno y, por lo general, es un cambio progresivo, es limitado y progresivo; lo trascendente de la presente investigación es determinar en qué medida esa adaptación al deber ser inmediata incidió en estrategias de actuación imprescindibles y de impacto positivo para que las empresas lograran su sobrevivencia, ya que podría implicar la pérdida de control sobre las acciones de gestión y esto no fue así. De aquí que la presente investigación se sustenta en la teoría de la contingencia, que establece que las organizaciones se deben adaptar a las fuentes externas de incertidumbre y complejidad (Lawrence y Lorsch, 1967; Lawrence y Dyer, 1983). Lo que aumenta la necesidad de procesamiento de la información y de acciones de cambio (Burton y Obel, 2004).

Con base en la argumentación anterior, el objetivo de la presente investigación fue determinar en qué medida la gestión del cambio influye en la colaboración virtual, en la transformación digital y en la agilidad estratégica organizacional ante la pandemia, cuyas interrogantes de investigación se presentan a continuación:

1. ¿Cuál es el efecto de la gestión del cambio en la colaboración virtual?

2. ¿Cuál es el efecto de la gestión del cambio en la transformación digital?

3. ¿Cuál es el efecto de la gestión del cambio en la agilidad estratégica organizacional?

El presente estudio contribuye a ampliar el conocimiento actual de la ciencia administrativa, estableciendo que la gestión del cambio influye sobre la colaboración virtual, sobre la transformación digital y sobre la agilidad estratégica organizacional ante escenarios críticos. El presente documento está estructurado de la siguiente forma: primero, se presenta una revisión de la literatura que argumenta las hipótesis de investigación. En segundo lugar, se describe el método de trabajo y los resultados estadísticos; y se finaliza con los hallazgos, discusión, limitaciones e implicaciones de la investigación. 


\section{$\underline{\text { Revisión de literatura e hipótesis }}$}

\section{Colaboración virtual, transformación digital y la gestión del cambio}

Aproximadamente la mitad de las empresas tenían más de $80 \%$ de sus empleados trabajando desde casa durante la primera etapa de la pandemia por la COVID-19 (a cinco meses de que se decretara emergencia en China y se extendiera el virus a todo el mundo; detectado el 7 de enero del año 2020 por científicos chinos llamado provisionalmente 2019-nCoV y posteriormente SARS-CoV-2) y estimaron aumentos sustanciales a largo plazo para el trabajo a distancia después de la pandemia. La necesidad de millones de trabajadores para dar respuesta a esta crisis ha acelerado el trabajo a distancia facilitado por las tecnologías de la conectividad y las comunicaciones (Kniffin, 2020), no sin dejar de considerar que el cambio ha sido costoso, consume tiempo y es inexacto (Andriole, 2017).

La pandemia de COVID-19 ha agilizado, de diversa maneras, la colaboración virtual en las organizaciones, ya que éstas han trasladado sus operaciones en línea utilizando herramientas virtuales (Baldwin, 2020). Es así que la pandemia alteró abruptamente las rutinas tradicionales de trabajar, y provocó una aceleración de las actividades que, de alguna manera, ya estaban en marcha y funcionando y que implicaban la migración del trabajo a entornos en línea o virtuales (Kniffin et al., 2020).

La crisis de COVID-19 ha generado el trabajo de colaboración virtual. Grandes sectores de la sociedad se encuentran en la actualidad confiando en los medios de comunicación virtuales para llevar a cabo las tareas de colaboración. Aunque es posible considerar que cuando no se habían construido relaciones de trabajo fuertes antes de la crisis, cuando no se había trabajado ni gestionado a distancia a través de medios de comunicación virtuales: la comunicación, el intercambio de conocimiento, el entendimiento común de normas objetivos y de tareas ha sido mucho más arduo (Caligiuri, 2020).

Pero se ha podido comprobar que esta colaboración virtual permite reducir los gastos, aumentar la productividad, mejorar los beneficios, mejorar el servicio al cliente, acceder a nuevos mercados, aumentar la productividad generando mayores beneficios, sin dejar de lado que también se perciben sentimientos de aislamiento y de falta de confianza (Heller, 2010; Cascio, 2020).

De manera específica, se requiere que las políticas empresariales existentes conlleven de la gestión del cambio y de la capacitación para logar la colaboración virtual (Cascio, 2020; Zeller, 2013) 
Es imperativo que la colaboración virtual deba estar alineada con la estrategia general de la organización y, por lo tanto, con el manejo del cambio (Hloma y Ortlepp, 2006). Las organizaciones deben reconocer que la comunicación virtual es sostenible, y que con los instrumentos tecnológicos, con las personas y con los procesos adecuados pueden lograr una alta calidad y satisfacción de los clientes (Heller, 2010).

La capacidad de adaptarse rápidamente a los cambios y de gestionarlo de la mejor manera es una capacidad importante, por lo tanto, ante esta necesidad, las organizaciones deben ser capaces de construir las habilidades necesarias para capitalizar las tendencias digitales. Al ser los cambios rápidos e intensos, es posible que las tecnologías digitales puedan transformar las organizaciones (Kane, Palmer, Phillips, Kiron, y Buckley, 2015). Diversos estudios han identificado que el uso de nuevas tecnologías y herramientas requiere de una gestión de cambio unida a nuevas formas de hacer negocios. Estos procesos de cambio derivado de las crisis pueden provocar cambios en los estilos de liderazgo (Stoker, Garretsen y Soudis, 2019) y, de esta manera, en los mecanismos para administrar el cambio. Las tecnologías digitales están haciendo que la ventaja competitiva tradicional se deteriore rápidamente y redefinan los modelos de negocio que han tenido éxito durante mucho tiempo, así como cambiar las formas de aprender (Bruch, Gerber, y Maier, 2005; Farias y Johnson, 2000).

La tecnología de la información, no es solo una respuesta a nivel funcional, sino es un impulsor fundamental de la creación y captura de valor organizacional. Las tecnologías digitales dan forma a la nueva infraestructura empresarial e influyen en la nueva lógica organizativa y en las pautas de coordinación dentro de las empresas y entre ellas (Bharadwaj, 2013). La transformación digital crea movimientos que desencadenan respuestas estratégicas de las organizaciones, generando creación de valor y al mismo tiempo gestionando cambios estructurales (Vial, 2019).

La transformación digital no tiene que ver fundamentalmente con la tecnología, sino con la estrategia (Rogers, 2016). La creación de capacidades para la transformación digital ha recibido una atención académica limitada y es ahora un contexto esencial para el estudio del cambio estratégico. Dado que la transformación digital deriva de un proceso de cambio muy complejo, se ha demostrado que esto requiere una serie de decisiones estratégicas calculadas e interdependientes con base en el manejo del cambio (Warner y Wäger, 2018).

La transformación digital se define como el grado de digitalización global de una empresa, que se refleja a través de: los artefactos digitales referentes a aplicaciones o contenidos multimedia 
con funciones y valores específicos integrados en productos o servicios digitales; de una plataforma digital que es un conjunto de servicios y arquitecturas generales compartidas; a través de una infraestructura digital de herramientas y sistemas tecnológicos digitales; de un modelo de negocio digital que crea valor impulsado por la tecnología digital de la empresa, y; por un modelo de gestión digital que implica la aplicación de tecnologías digitales en el sistema de gestión de la organización, como la adopción de sistemas de oficina inteligentes. Así también la digitalización puede medirse distinguiendo los negocios en línea de los negocios fuera de línea; de aquí que de acuerdo con el avance en esta digitalización existan diversos niveles de transformación digital (Guo et al., 2020; Biswas y Burman, 2009).

Son diversos los estudios que han determinado la importancia de la gestión del cambio como estrategias que inciden en estrategias funcionales en las organizaciones (Fiss y Zajac, 2006; Helfat et al., 2007; Lüscher y Lewis, 2008; MacKay y Chia, 2013).

La gestión del cambio implica actividades de motivación del cambio tales como ayudar a los empleados a superar la resistencia mediante la creación de ambientes que vislumbren la necesidad del cambio y que derive de identificar procesos decisorios; consiste en crear una visión vinculada orientada y justificada con las actividades esenciales de la dirección hacia la descripción del futuro deseado; consiste en obtener el apoyo necesario para el cambio; asimismo implica gestionar la transición del estado actual al deseado, lo cual requiere de planeación de actividades y de acciones estructurales y de sostener las acciones impulsoras de cambio hasta generar el cambio a un término exitoso mediante competencias y habilidades que refuercen las conductas organizacionales necesarias (Cummings y Worley, 2007).

\section{La agilidad estratégica organizacional ante la pandemia y la gestión del cambio}

La agilidad organizacional se considera una respuesta a los cambios continuos e impredecibles y, por lo tanto, es particularmente necesaria y eficaz en un entorno en constante cambio, volátil e impredecible (Teece et al., 2016 y Walter, 2020).

El cambio organizativo es esencial para la competitividad a corto plazo y la supervivencia a largo plazo, pero plantea enormes desafíos de gestión (Lüscher y Lewis, 2008). La gestión del cambio se ha convertido en la responsabilidad última de la dirección, pues las organizaciones continuamente se enfrentan a la necesidad de la implementación de algún tipo de cambio (Kanter, Stein y Jick, 1992) y tienen el reto de captar un cambio que no diseñaron (Balogun y Johnson, 
2004). La agilidad organizacional permite adaptar y captar capacidades de cambio rápido y flexibles y gestionar eficazmente los cambios externos e internos inevitables con la finalidad de anticipar o responder a los cambios de manera oportuna y con facilidad (Van Oosterhout et al., 2006). Por ello, los altos directivos tratan de comprender la dinámica externa y con base en ella, inician un cambio organizativo, estas iniciativas de procesos de cambio ayudan a la comprensión del contexto y permiten generar un sentido de orden (McKinley y Scherer, 2000; Lüscher y Lewis, 2008).

Las organizaciones se enfrentan al reto de cómo adaptarse al creciente ritmo de cambio en mundos más complejos. Los contextos de incertidumbres o de sorpresa impulsan procesos de enseñanza dirigidos hacia la adaptabilidad de las organizaciones y se requiere que éstas sean ágiles estratégicamente (Woods, 2020).

La agilidad estratégica es la capacidad de una organización para ajustar continuamente la dirección estratégica y desarrollar formas innovadoras de crear valor; es la capacidad de la dirección de percibir y responder de forma constante y rápida a un entorno cambiante realizando intencionadamente movimientos estratégicos y adaptando consecuentemente la configuración organizativa necesaria para una implementación exitosa (Weber y Tarba, 2014); es la capacidad de reconocer y aprovechar rápidamente las oportunidades, cambiar de dirección y evitar las colisiones (McCann, 2004).

La agilidad estratégica constituye la capacidad de las empresas para asumir compromisos estratégicos y, al mismo tiempo, gestionar y ajustarse a los cambios continuos causados por las crecientes discontinuidades y perturbaciones estratégicas (Doz y Kosonen, 2008; Ivory y Brooks, 2018)

Ante un entorno de crisis turbulenta, las acciones y las respuestas de las organizaciones deben implicar la modificación o la adaptación a ésta y pueden ser de dos tipos: respuestas de emergencia a corto plazo para la supervivencia y respuestas estratégicas a largo plazo para el desarrollo (Guo et al., 2020). Por ello, la agilidad estratégica de las organizaciones ante la pandemia implicó mecanismos de recuperación de la producción, a través de la adopción del teletrabajo en línea, de la optimización de los modelos de negocio para captar las nuevas necesidades de los clientes, de la inversión en innovación tecnológica y de la diversificaron en nuevas áreas de negocios (Wenzel, Stanske y Lieberman, 2020; Guo et al., 2020).

De acuerdo con la argumentación anterior, surgen las siguientes hipótesis: 
H1: La gestión del cambio tiene un efecto positivo en la colaboración virtual.

H2: La gestión del cambio tiene un efecto positivo en la transformación digital.

H3: La gestión del cambio tiene un efecto positivo en la agilidad estratégica organizacional ante la pandemia

\section{Método}

\section{Diseño de investigación}

El diseño de investigación (Leedy y Ormrod, 2001) será de tipo transversal -transeccional- y no experimental, debido a que la recolección de datos se realizó en un solo momento y no habrá intervención en las variables de estudio. De acuerdo con la organización del estudio, será un estudio correlacional, ya que incluirá técnicas de correlación y regresión (Hair, Anderson, Tatham y Black, 2008).

\section{Muestra y caracterización}

Con la finalidad de cumplir con el objetivo de la investigación, este estudio empírico llevó a cabo un proceso de muestreo no probabilístico (por conveniencia-sujetos voluntarios) a 109 empresas de México; cuya unidad de análisis fue en su mayoría mandos directivos y medios. Se garantizó la confidencialidad y el anonimato de los participantes. Las empresas fueron elegidas buscando las unidades más representativas y convenientes para el estudio.

Respecto a la caracterización de las empresas, 80 (73.4\%) pertenecen al giro de productos; $6(5.5 \%)$ son del giro comercial o intermediaria y $23(21.1 \%)$ del giro de servicios.

Respecto del tamaño de la empresa, 55 (50.5\%) empresas son grandes; 22 (20.2\%) son medianas, $19(17.4 \%)$ son pequeñas y $13(11.9 \%)$ son microempresas.

Respecto del sector de la empresa, $19.3 \%$ son del sector automotriz; $11.9 \%$ del sector alimentación; $8.3 \%$ del sector comercio; $5.5 \%$ del sector farmacéutico; $4.6 \%$ del sector de la construcción; $3.7 \%$ del sector educación; $1.8 \%$ del sector agricultura y $44 \%$ pertenecen a otro sector.

Respecto a la caracterización de los participantes, se observó una muestra en donde la mayoría son hombres, es decir, 60.6\% fueron hombres y 39.4 mujeres; el mayor número de participantes osciló entre una edad de 31 a 40 años (55.5\%); 22\% tuvieron entre 20 y 30 años, $13.8 \%$ de 41 a 50 años; la minoría (9.2\%) tiene más de cincuenta años. En lo referente a la jerarquía 
laboral (puesto), $49.5 \%$ fueron mandos medios; $31.2 \%$ mandos o directivos, y la minoría fue personal nivel staff $10.1 \%$ y operativo $9.2 \%$. En cuanto a la antigüedad laboral, $11.9 \%$ dijo tener menos de 1 año, $45.9 \%$ de 1 a 5 años; $23.9 \%$ de 6 a 10 años; $18.3 \%$ más de diez años.

Referente al grado de estudios o escolaridad, la mayoría cuenta con estudios de posgrado (51.4), 47.7\% manifestó ser licenciado.

Respecto al tipo de contrato, $89.9 \%$ cuenta con contrato definitivo, $5.5 \%$ es personal outsourcing y $4.6 \%$ tiene contrato temporal.

\section{Instrumento de recolección de datos}

La estrategia de investigación (Creswell, 2009; Leedy y Ormrod, 2001) fue la encuesta, mediante la aplicación de un cuestionario en línea durante las tres últimas semanas de septiembre y la primera de octubre. Los datos se recolectaron en empresas mexicanas.

El instrumento se integró por dos secciones: la primera contiene datos de las empresas (giro de la empresa, tamaño de la empresa, sector de la empresa) y datos demográficos y laborales de los respondientes (nivel jerárquico, edad, nivel de estudios, antigüedad, tipo de contrato, género) $\mathrm{y}$, la segunda contiene los reactivos para medir las variables objeto de estudio. Todos los reactivos se plantearon en escala likert de siete opciones que va de 1(totalmente en desacuerdo) a 7 (totalmente de acuerdo).

El instrumento fue construido a partir de las contribuciones teóricas de diversos autores Guo et al. (2020); Caligiuri et al. (2020) y Cummings y Worley (2013).

La variable dependiente colaboración virtual, se basó en Caligiuri et al. (2020), conformada por cuatro reactivos o ítems y fue medida de manera unidimensional; la variable dependiente de transformación digital se soportó por Guo et al. (2020) conformada por cuatro reactivos o ítems y fue unidimensional. La variable dependiente agilidad organizacional estratégica ante la pandemia se conformó por cuatro reactivos referentes a las estrategias recuperación de la producción sustentada por Guo et al. (2020) y Wenzel, Stanske y Lieberman (2020).

La variable independiente gestión del cambio se basó en Cummings y Worley (2013), operacionalizada con seis reactivos que derivaron de la conceptualización del desarrollo organizacional y el cambio.

Para ayudar a perfeccionar el cuestionario, se realizó una validación de contenido a través de expertos, cuyas sugerencias fueron incorporadas en la segunda revisión del mismo. 


\section{Métodos de estimación}

Para la ponderación de las variables, se utilizó el método de componentes principales con una rotación varimax ortogonal para establecer la estructura factorial del instrumento (Nunnally y Bernstein, 1995), con la finalidad de hacer una evaluación de las diferentes escalas de las dimensiones de cada una de las variables latentes o constructos.

En el análisis factorial exploratorio cada variable se explica por su ponderación en cada factor con la finalidad de representar, lo mejor posible, las variables en un número reducido de factores, es decir, los factores referidos a dimensiones subyacentes de los datos, en donde cada variable tiene una carga sobre cada factor (Hair et al., 2008).

No se encontraron problemas de dimensionalidad en ninguna variable. Considerando que todos los datos fueron recolectados del mismo instrumento de medida, fue necesario comprobar la presencia del sesgo de la varianza del método común a través del test de un factor de Harman (Konrad y Linnehan, 1995). Los resultados del análisis factorial exploratorio revelan que las variables no pertenecen a un único factor y, por lo tanto, se puede atribuir que la varianza de las variables del estudio se debe a los constructos que se evalúan y no al método de evaluación (Konrad y Linnehan, 1995).

En las estructuras factoriales obtenidas, todos los ítems presentaron cargas o saturación superiores a 0.5, criterio a partir del cual se consideran aceptables (Castañeda, Cabrera y Navarro, 2010), con una varianza explicada del $44.86 \%$.

Además, en este análisis factorial exploratorio se evaluó el índice de adecuación de la muestra de Kaiser-Meyer-Oklin (KMO); el cual se valora dentro de un rango de 0 y 1, considerando que un valor aceptable se ubica entre 0.80 y 0.90. La prueba de esfericidad de Bartlett, que contrasta la hipótesis nula de que la matriz de correlaciones es una matriz de identidad, resultó significativa, con $\mathrm{p}=0.00$, lo que deriva en una adecuación muestral.

En la Tabla 1 se puede observar los resultados del análisis factorial exploratorio de las variables en estudio. 
Tabla 1. Matriz de componente rotado.

Table 1. Rotated component array.

\begin{tabular}{|c|c|c|c|c|}
\hline \multirow[b]{2}{*}{ Reactivo/Ítem } & \multicolumn{4}{|c|}{ Componente } \\
\hline & 1 & 2 & 3 & 4 \\
\hline R6 & .850 & & & \\
\hline $\mathrm{R} 2$ & .740 & & & \\
\hline $\mathrm{R} 4$ & .712 & & .479 & \\
\hline $\mathrm{R} 1$ & .697 & & & \\
\hline R3 & .687 & & & \\
\hline R5 & .598 & & .468 & \\
\hline R9 & & .883 & & \\
\hline $\mathrm{R} 10$ & & .849 & & \\
\hline R7 & & .764 & & \\
\hline R8 & & .731 & & \\
\hline R11 & & .509 & & \\
\hline R17 & & & .732 & \\
\hline $\mathrm{R} 18$ & & & .633 & \\
\hline R16 & & & .603 & \\
\hline $\mathrm{R} 15$ & & .481 & .565 & \\
\hline $\mathrm{R} 13$ & & & & .776 \\
\hline $\mathrm{R} 12$ & & & & .760 \\
\hline R14 & & & & .748 \\
\hline
\end{tabular}

Método de extracción: análisis de componentes principales.

Método de rotación: Varimax con normalización Kaiser.

Extraction method: principal component analysis.

Rotation method: Varimax with Kaiser normalization.

Se empleó el análisis multivariante, específicamente la modelación de ecuaciones estructurales (SEM). Se utilizó el paquete estadístico AMOS (Analysis of Moment Structures) versión 22. Se obtuvo la validez convergente (fiabilidad de constructo) y la varianza media extraída del modelo de medición.

\section{Análisis de normalidad y multicolinealidad}

Como se puede observar en la Tabla 2, al verificarse el supuesto de normalidad mediante los valores de la asimetría y la curtosis, los valores permitidos se encuentran entre \pm 2 (Pérez, 2008). Con la asimetría y la curtosis se comprueba las variables de la investigación presentan una distribución normal. 
Tabla 2. Normalidad.

Table 2. Normality.

\begin{tabular}{|ccc|}
\hline Variables & Asimetría & Curtosis \\
\hline Gestión del cambio & -.54 & 1.30 \\
Colaboración virtual & -.23 & 1.30 \\
Transformación digital & -.88 & 1.59 \\
Agilidad estratégica organizacional ante la pandemia & -.69 & 1.09 \\
\end{tabular}

Con respecto a la multicolinealidad: el valor mostrado del factor de inflación de la varianza (FIV) muestra valores por debajo de 3, y la tolerancia mostrada-para cada una de las variables independientes se encuentra en un nivel aceptable (Martín, Cabero y De Paz, 2008).

\section{$\underline{\text { Resultados }}$}

Derivado de que se entrevistó a personal de varios sectores industriales, y con la finalidad de saber si hubo cambios en los sectores se aplicó la prueba estadística de comparación de medias ANOVA de un factor mediante la prueba Tukey, y no se encontró significancia significativa entre las variables de investigación: en los tres casos la significancia fue mayor a 0.08.

La Tabla 3 reporta las correlaciones para las variables utilizadas en este estudio. Las correlaciones entre los constructos fueron de medias a altas (0.41 a 0.71). Todas las correlaciones son estadísticamente significativas. El alfa de Cronbach para la variable gestión del cambio es 0.90, para la variable colaboración virtual es 0.76 , para la variable transformación digital es 0.90 y para la variable agilidad estratégica organizacional ante la pandemia es 0.76 . De acuerdo con los índices obtenidos, se observa consistencia interna de los reactivos de cada variable.

Tabla 3. Estadística descriptiva y correlaciones $(n=109)$.

Table 3. Descriptive statistics and correlations $(n=109)$.

\begin{tabular}{|c|c|c|c|c|c|c|}
\hline Variable & Media & $\begin{array}{l}\text { Desviación } \\
\text { Estándar }\end{array}$ & $\begin{array}{c}\text { Gestión } \\
\text { del } \\
\text { cambio }\end{array}$ & $\begin{array}{c}\text { Colaboración } \\
\text { virtual }\end{array}$ & $\begin{array}{c}\text { Transformación } \\
\text { digital }\end{array}$ & $\begin{array}{c}\text { Agilidad } \\
\text { estratégica } \\
\text { organizacional } \\
\text { ante la pandemia }\end{array}$ \\
\hline Gestión del cambio & 5.2 & 1.03 & $(.90)$ & & & \\
\hline Colaboración virtual & 4.9 & 1.04 & $.71 * *$ & $(.76)$ & & \\
\hline Transformación digital & 5.3 & 1.2 & $.41 * *$ & $.43 * *$ & $(.90)$ & \\
\hline $\begin{array}{c}\text { Agilidad estratégica } \\
\text { organizacional ante la } \\
\text { pandemia }\end{array}$ & 4.9 & 1.2 & $.53 * *$ & $.42 * *$ & $.62^{* *}$ & (.76) \\
\hline $\begin{array}{l}\text { a: ** La correlación es sig } \\
\text { Los datos entre parénte }\end{array}$ & $\begin{array}{l}\text { iva al } n \\
\text { la conf }\end{array}$ & $\begin{array}{l}\text { de } 0.01(2-c \\
\text { lidad de la v }\end{array}$ & (alf & onbach) & & \\
\hline
\end{tabular}


La Tabla 4 reporta las cargas factoriales y la varianza explicada de las variables. Todas las cargas factoriales de las variables latentes son significativas y consistentes con los coeficientes estandarizados de sus reactivos. Esta tabla también resume la varianza explicada de las medidas observadas con respecto a sus constructos. La mayoría de estos reactivos tienen valores altos de $\mathrm{R}^{2}$, lo que sugiere una confiabilidad aceptable.

Tabla 4. Cargas factoriales y R2.

Table 4. Factor Loadings and R2.

\begin{tabular}{|c|c|c|c|c|}
\hline Variable y sus reactivos & $\begin{array}{c}\text { Coeficientes } \\
\text { estandarizados }\end{array}$ & $\begin{array}{l}\text { Error } \\
\text { estándar }\end{array}$ & $\begin{array}{c}\mathbf{P} \\
\text { significativa }\end{array}$ & $\mathbf{R}^{2}$ \\
\hline \multicolumn{5}{|l|}{ Gestión del cambio } \\
\hline $\mathrm{X} 1$ & 0.76 & & $* * *$ & .58 \\
\hline $\mathrm{X} 2$ & 0.82 & .12 & $* * *$ & .67 \\
\hline $\mathrm{X} 3$ & 0.71 & .12 & $* * *$ & .50 \\
\hline $\mathrm{X} 4$ & 0.81 & .12 & $* * *$ & .66 \\
\hline $\mathrm{X} 5$ & 0.72 & .12 & $* * *$ & .52 \\
\hline X6 & 0.88 & .12 & $* * *$ & .77 \\
\hline \multicolumn{5}{|l|}{ Transformación digital } \\
\hline $\mathrm{X} 7$ & 0.78 & & $* * *$ & .60 \\
\hline $\mathrm{X} 8$ & 0.75 & .11 & $* * *$ & .56 \\
\hline $\mathrm{X} 9$ & 0.89 & .09 & $* * *$ & .80 \\
\hline $\mathrm{X} 10$ & 0.70 & .10 & $* * *$ & .76 \\
\hline \multicolumn{5}{|l|}{ Colaboración virtual } \\
\hline $\mathrm{X} 11$ & 0.39 & & $* * *$ & .15 \\
\hline $\mathrm{X} 12$ & 0.73 & .50 & $* * *$ & .54 \\
\hline $\mathrm{X} 13$ & 0.86 & .50 & $* * *$ & .74 \\
\hline $\mathrm{X} 14$ & 0.70 & .52 & $* * *$ & .59 \\
\hline \multicolumn{5}{|l|}{$\begin{array}{c}\text { Agilidad estratégica } \\
\text { organizacional ante la } \\
\text { pandemia }\end{array}$} \\
\hline $\mathrm{X} 15$ & 0.72 & & $* * *$ & .51 \\
\hline $\mathrm{X} 16$ & 0.74 & .23 & $* * *$ & .55 \\
\hline $\mathrm{X} 17$ & 0.72 & .23 & $* * *$ & .52 \\
\hline $\mathrm{X} 18$ & 0.51 & .27 & $* * *$ & .26 \\
\hline${ }^{* * * *}(\mathrm{p}<0.001)$ & & & & \\
\hline
\end{tabular}

En la Tabla 5 se observa el estadístico razón de verosimilitud chi cuadrada $X^{2}$, el cual presenta un valor de 235.59, lo cual significa que las matrices observadas y estimadas difieren considerablemente. Sin embargo, dada la sensibilidad estadística de esta medida al tamaño de la 
La gestión del cambio, la colaboración virtual y la agilidad estratégica organizacional de empresas mexicanas ante los impactos por el COVID-19

muestra, se complementó con otras medidas de calidad del ajuste. Con el RMSEA se observa que la discrepancia por grado de libertad entre las matrices de entrada estimadas y las observadas son aceptables ya que tiene un valor de 0.080. En cuanto al NFI, se observa que este índice es adecuado pues presenta un valor de .88. El CFI y el IFI presentan una buena calidad del ajuste, ya que sus valores son de .90 . El GFI presenta un valor .88. Por lo anterior, se considera que el modelo de investigación se ajusta a los datos observados de manera adecuada.

En la Tabla 5 también se observa la validez convergente (fiabilidad del constructo). Para la variable gestión del cambio es de 0.90, para la variable colaboración virtual es de 0.77 , para la variable transformación digital es de 0.89 y para la variable agilidad estratégica organizacional ante la pandemia es de 0.76 . Los valores evidencian una fiabilidad del constructo con índices por encima de los criterios de evaluación (0.6) (Bagozzi y Yi, 1988). En cuanto a la varianza extraída de los constructos, en dos de ellos (colaboración virtual y agilidad estratégica organizacional ante la pandemia) los resultados son cercanos al punto de corte mínimo sugerido del 0.50 y dos variables gestión del cambio y transformación digital rebasan dicho valor mínimo (Fornell y Larcker, 1981; Hair et al., 2008). Por lo anterior, el modelo de medición es adecuado.

Tabla 5. Validez convergente, Varianza media extraída y Ajuste del modelo.

Table 5. Convergent Validity and Goodness of fit measures.

\begin{tabular}{|c|c|c|}
\hline Variable & 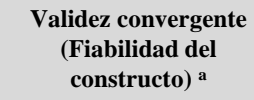 & $\begin{array}{c}\text { Varianza media } \\
\text { extraída }^{b}\end{array}$ \\
\hline Gestión del cambio & & 0.61 \\
\hline Colaboración virtual & & 0.47 \\
\hline Transformación digital & & 0.67 \\
\hline \multicolumn{2}{|l|}{$\begin{array}{c}\text { Agilidad estratégica organizacional ante la } \\
\text { pandemia }\end{array}$} & 0.45 \\
\hline \multicolumn{3}{|c|}{ Medidas de ajuste del modelo y de los modelos de medida de las variables } \\
\hline $\mathrm{X}^{2}$ & 236.59 & \\
\hline $\mathrm{X}^{2} / \mathrm{GL}$ & 1.82 & \\
\hline $\begin{array}{l}\text { RMSEA (error de aproximación cuadrático } \\
\text { medio) }\end{array}$ & .080 & \\
\hline NFI (índice de ajuste normado) & .88 & \\
\hline CFI ( índice de ajuste comparado) & .90 & \\
\hline IFI (índice de ajuste incremental) & .90 & \\
\hline GFI (índice de bondad de ajuste) & .88 & \\
\hline \multicolumn{3}{|c|}{$\begin{array}{l}\text { a Validez convergente es calculada utilizando la fórmula: (Suma de las ponderaciones estandarizadas) })^{2} \text { ( }(\text { Suma de las } \\
\text { ponderaciones estandarizadas) }{ }^{2}+(\text { Suma del error de medida del indicador) (Hair, et al, 2008). } \\
\text { b Varianza media extraída es calculada utilizando la fórmula: (Suma de los cuadrados de las ponderaciones estandarizadas) / } \\
\text { (Suma de los cuadrados de las ponderaciones estandarizadas + Suma del error de medida) (Hair, et al, 2008). }\end{array}$} \\
\hline
\end{tabular}


Los resultados del modelo estructural utilizado para apoyar las hipótesis se muestran en la Fig. 1. Todas las cargas factoriales fueron estadísticamente significativas $(\mathrm{p}<0.001)$ y mayores a 0.38 , las cuales pueden ser interpretadas para evaluar la fuerza y la significancia de las relaciones del modelo.

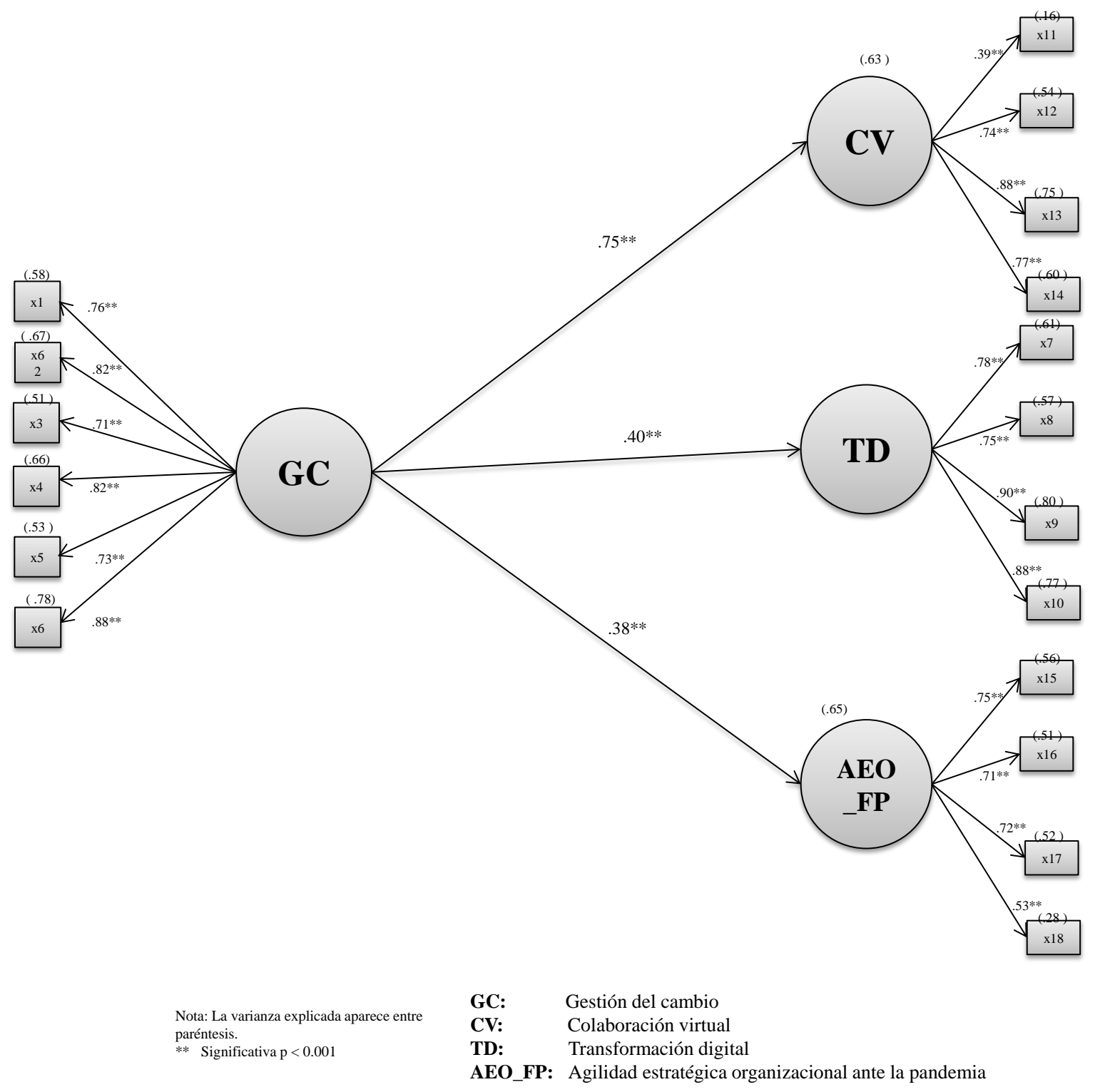

Fig. 1. Modelo estructural.

Fig. 1. Structural model. 
Como se muestra en la Fig. 1, la trayectoria estructural de la gestión del cambio sobre la colaboración virtual es positiva y estadísticamente significativa $(\gamma=0.75 ; \mathrm{p}<0.001)$ Este resultado provee un apoyo para la hipótesis 1.

La trayectoria estructural de la gestión del cambio sobre la transformación digital $(\gamma=0.40$; p $<0.001)$ es positiva y estadísticamente significativa. Se puede considerar que la gestión del cambio tiene un efecto positivo sobre la transformación digital, resultado que apoya la hipótesis 2 .

La trayectoria estructural de la gestión del cambio sobre la agilidad estratégica organizacional ante la pandemia $(\gamma=0.38 p<0.001)$ es positiva y estadísticamente significativa. Se puede considerar que la gestión del cambio tiene un efecto positivo sobre la agilidad estratégica organizacional ante la pandemia, resultado que apoya la hipótesis 3 . Asimismo se observa en la figura 1 que la varianza explicada del modelo es de .63, .16 y .65

El diseño de esta investigación fue transeccional, es así que no es posible establecer una relación de causalidad entre las variables en estudio, ya que la interpretación de la causalidad en el mundo real no se garantiza (Kline, 2005). Sin embargo, es posible establecer con estos resultados una discusión acerca de la influencia de la gestión del cambio en la colaboración virtual, en la transformación digital y en la agilidad estratégica organizacional ante la pandemia.

\section{Discusión de resultados}

El objetivo de la investigación fue determinar en qué medida la gestión del cambio influye en la colaboración virtual, en la transformación digital y en la agilidad estratégica organizacional ante la pandemia en empresas mexicanas como respuesta ante la crisis COVID-19. Ante este entorno emergente las empresas replantearon sus estrategias para enfrentarse a la crisis generada por los impactos de la COVID-19.

La presente investigación nos lleva a identificar la importancia de la necesidad de adaptación inmediata (no siempre planeada) mediante capacidades de gestión de cambio y de administrarlo de la mejor manera, ya que es una capacidad importante y fundamental para mantener y a la vez, transformar a las organizaciones; al ser los cambios rápidos e intensos, es necesario que se gestione este proceso e incidir así para que las organizaciones sean competitivas a corto plazo y sobrevivan a largo plazo.

Bajo esta argumentación y desde la teoría de la contingencia que establece que las organizaciones se deben adaptar a las fuentes externas de incertidumbre y de complejidad (en este 
caso la crisis por la COVID-19) y donde los factores de contingencia son factores de contexto que moderan la relación entre un sistema organizativo y su rendimiento (Hamman, 2017), los hallazgos de la investigación lo comprueban e indican que la gestión del cambio predice la colaboración virtual, la transformación digital y la agilidad estratégica organizacional.

La teoría de la contingencia explica la necesidad de la adaptabilidad organizacional derivado de que el ambiente ya sea interno o externo (en este caso, externo: la pandemia) siempre cambia, de aquí que las organizaciones deben desarrollar estrategias continuas para cualquier cambio que tengan que implementar, de acuerdo con Labarca (2008), deberán responder al ambiente cambiante y hacerle frente. Según Mintzberg (1990), la estrategia fundamental es sostener la estabilidad y, periódicamente, aunque de manera imprevista deben reconocer la necesidad de una transformación, tratando de manejar ese proceso imprevisto y atípico sin destruir la organización. De aquí que, la pandemia y sus repercusiones al no tenerse planeadas, las organizaciones no actuaron por una ideología de cambio, sino que la situación lo forzó, las estrategias dependieron del momento y del contexto; por ello, al enfrentarse a esta situación imprevista, las organizaciones debieron motivar el deseo de este proceso de manera inmediata, se construyeron soportes de agentes de cambio, se construyó una ideología de cambio inmediato, se planearon actividades a través del desarrollo de estrategias de compromiso del personal, lo que implicó, entonces, que las organizaciones conllevaran exitosamente la colaboración virtual, la transformación digital y la agilidad organizacional estratégica. De manera específica, esta investigación contribuye teóricamente a determinar que la gestión del cambio es un factor incidente en estrategias necesarias inmediatas estratégicas. De aquí que las organizaciones que enfrentan el medio ambiente sin tener la capacidad de generar una adaptabilidad a los cambios quedan inoperantes (Shipp y Jansen, 2011).

La evidencia empírica encontrada en la literatura ayuda a confirmar los resultados esperados de estas relaciones (Heller, 2010; Kane, Palmer, Phillips, Kiron, \& Buckley, 2015; Bharadwaj, 2013; Vial, 2019; Warner y Wäger, 2018; McKinley y Scherer, 2000; Lüscher y Lewis, 2008; Weber y Tarba, 2014; McCann, 2004; Doz y Kosonen, 2008; Ivory y Brooks, 2018; Lüscher y Lewis, 2008; Van Oosterhout et al., 2006; Teece, et al., 2016 y Walter, 2020; Heller, 2010; Hloma y Ortlepp, 2006).

La pandemia mundial generó una profunda crisis económica y se ha requerido hacer frente a las catastróficas consecuencias socioeconómicas, humanitarias y de derechos humanos. 
Asimismo, las organizaciones requirieron adoptar procesos que hicieran frente y dieran respuestas a estos escenarios caóticos derivados de la crisis COVID-19 y, además, se debió dar una certera resolución colaborativa de problemas. Por ello, empresas mexicanas detonaron la colaboración virtual mediante estrategias de coincidencia del tipo de tecnología de la información y de las comunicaciones con la tarea principal a desarrollar; desarrollaron las habilidades de colaboración virtual a través de una capacitación formal abarcando las TIC's; la integración y la colaboración en los equipos virtuales permitieron a los empleados desarrollar una mejor comprensión compartida de sus tareas, objetivos y normas sociales y les permitieron construir lazos sociales más fuertes y una identidad de equipo compartida.

Las empresas mexicanas tuvieron que fortalecer la aplicación de las tareas de oficina en línea, mejorar la digitalización de los canales de la cadena de suministro, adoptar plataformas digitales y plataformas de comunicación digital, así como infraestructuras digitales.

Ante la pandemia, las empresas mexicanas, para ser ágiles estratégicamente adoptaron el teletrabajo en línea, optimizaron los modelos de negocio para captar las nuevas necesidades de los clientes, invirtieron en innovación tecnológica y se diversificaron en nuevas áreas de negocios. Enfocándose en la gestión del cambio, el análisis empírico de esta investigación ha puesto de manifiesto la importancia de éste para generar capacidades necesarias que las organizaciones requieren ante respuestas prontas y necesarias para garantizar su supervivencia y competitividad. Con estos resultados, este estudio aporta un valor teórico al conocimiento al confirmar una relación positiva y significativa de la gestión del cambio en diversas capacidades necesarias de las organizaciones ante la crisis, lo cual se sustenta y se predice por la teoría de la contingencia que establece que las organizaciones se deben adaptar a las fuentes externas de incertidumbre y complejidad.

\section{Limitaciones y sugerencias}

Aun y cuando este estudio mostró un efecto positivo y significativo de la gestión del cambio (ante la crisis por la COVID-19) en la colaboración virtual, en la transformación digital y en la agilidad estratégica organizacional, se basó en datos de corte transversal. Sin embargo, futuras investigaciones deben incorporar un estudio longitudinal que pruebe los constructos en diferentes tiempos y demuestre las relaciones sustentadas en la teoría. 
Asimismo, es fundamental considerar que derivado de que 50\% de la comunicación de contenido emocional es trasmitida por la expresión facial, el resto es por el tono de voz y por lo que se dice, las emociones de los empleados que han tenido que hacer frente a la transformación digital y a la colaboración virtual están en desventaja por la ausencia de interacción física, ya que el reflejo de las emociones es parte de la interacción y a su vez éstas pueden llegar a influir en el rendimiento. De aquí que otros estudios deben perfilarse hacia la investigación concreta de las implicaciones de la colaboración virtual respecto a las sensaciones de los empleados y su incidencia, las organizaciones deberán preocuparse por la motivación diaria de los empleados, ya que al perder los contactos sociales y laborales se puede perder, incluso, el crecimiento profesional (Cañadas, 2017). Además, la transformación digital y la colaboración virtual implican un equilibrio entre la vida y el trabajo, implican un equilibrio con respecto a las oportunidades de promoción, la equidad interna y externa de sus salarios y el balance entre familia-labor; no obstante, también es visible que los asuntos urgentes y los requerimientos de trabajo puedan en algún momento tomar tiempo de la familia y el descanso, desencadenado cierto nivel de estrés. Otra implicación es que uno de los principales obstáculos para implementar el teletrabajo es la percepción de los altos costos frente a pocos beneficios. Así, es visible que las fuerzas que favorecieron el cambio para implementar el teletrabajo, fueron en primer lugar la flexibilidad, seguido de la iniciativa y en tercer lugar la confianza. Pero las fuerzas que obstaculizaron pudieron ser la falta de incentivos y el miedo al fracaso (García, Guevara y Mella, 2007).

\section{Implicaciones prácticas}

Este estudio proporciona una implicación práctica referente a las estrategias inmediatas que realizaron empresas mexicanas ante la crisis por la COVID-19; entre ellas, la necesidad de manejar eficazmente el cambio mediante diversas estrategias para que la colaboración virtual, la transformación digital y la agilidad estratégica organizacional pudieran funcionar de la mejor manera.

\section{$\underline{\text { Referencias }}$}

ALAMPYME (2020). https://integraa.org/2020/03/asociacion-latinoamericana-de-microspequenos-y-medianos-empresarios-alampyme-por-crisis-estan-en-riesgo-de-cerrar-100mil-mipymes/ 
Andriole, S. J. (2017). Five myths about digital transformation. MIT Sloan Management Review, 58(3), 20-22. Disponible en https://sloanreview.mit.edu/article/five-myths-about-digitaltransformation/

Bagozzi, R.P y Yi, Y. (1988). On the evaluation of Structural Equation Models, Journal of the $\begin{array}{llllll}\text { Academy of } & \text { Marketing } & \text { Science, } & 16 & \text { (1), } & \text { 74-94. }\end{array}$ DOI: $10.1007 / \mathrm{BF} 02723327$

Baldwin, S. (2020). Watch Out, Corporates: Canada's Charitable Sector, Once Reluctant to Embrace Digital Transformation, is Now Competing for its Share of Eyeball Time. Disponible en: https://thephilanthropist.ca/2020/05/watch-out-corporates-canadascharitable-sector-once-reluctant-to-embrace-digital-transformation-is-now-competing-forits-share-of-eyeball-time/

Balogun, J., y Johnson, G. (2004). Organizational restructuring and middle manager sensemaking. $\begin{array}{lllll}\text { Academy of } & \text { Management }\end{array}$ DOI: $10.2307 / 20159600$

Bharadwaj, A. (2013). Digital business strategy: Toward a next generation of insights. MIS Quarterly. 37 (2), 471-482. Disponible en: https://ssrn.com/abstract=2742300

Biswas, D., y Burman, B. (2009). The effects of product digitalization and price dispersion on search intentions in offline versus online settings: The mediating effects of perceived risks. Journal of Product \& Brand Management, 18(7), 477-486. DOI: $10.1108 / 10610420910998208$

Bruch, H., Gerber, P. y Maier, V. (2005). Strategic change decisions: doing the right change right. Journal of Change Management, 5 (1), 97-107. https://doi.org/10.1080/14697010500067390

Caligiuri, P. et al. (2020). International HRM insights for navigating the COVID-19 pandemic: Implications for future research and practice. Journal of International Business Studies, 51, 697-713. DOI: $10.1057 / \mathrm{s} 41267-020-00335-9$

Cañadas, F. (2017). Teletrabajo: Revisión teórica y análisis jurídicolaboral. Granada, España: Universidad de Granada.

Cascio, W.F. (2020) Managing a virtual workplace. Academy of Management Perspectives. 14(3): 81-90. https://doi.org/10.5465/ame.2000.4468068 
Castañeda, M.B., Cabrera, A.F., y Navarro, Y. (2010). Procesando datos y análisis estadísticos utilizando SPSS. Brasil: Edipucrs.

Creswell, J. (2009). Research Design. Qualitative, Quantitative, and Mixed Methods Approaches. London: Sage Publications.

Cummings, T. G. y Worley, C. G. (2013). Organization development \& Change. USA: SouthWestern, Cengage Learning.

Doz, Y., y Kosonen, M. (2008). The dynamics of strategic agility: Nokia's rollercoaster experience. California Management Review, 50(3), 95-118. DOI: $10.2307 / 41166447$

Farias, G. y Johnson, H. (2000). Organizational development and change management: setting the record straight/response to Farias and Johnson's commentary. Journal of Applied Behavioral Science, 36 (3), 376-379. https://doi.org/10.1177/0021886300363007

Fiss, P.C. y Zajac, E.J. (2006). The symbolic management of strategic change: sensegiving via framing and decoupling. Academy of Management Journal, 49 (6), 1173-1193. https://doi.org/10.5465/AMJ.2006.23478255

Fornell, C. y Larcker, D. (1981). Evaluating Structural Equations Models with Unobservable Variables and Measurement Error, Journal of Marketing Research, 27, 39-50. DOI: $10.2307 / 3151312$

García, R. E., Guevara, M. E. y Mella, L. (2008). Calidad de vida laboral de teletrabajadores, en entornos de trabajo que utilizan las tecnologías de la información y la comunicación en la República Dominicana. Ciencia y Sociedad, XXXIII(3), 442-461. Disponible en: https://www.redalyc.org/pdf/870/87011545007.pdf

Guo, H., Yang, Z., Huang, R. y Guo, A. (2020). The digitalization and public crisis responses of small and medium enterprises: Implications from a COVID-19 survey. Frontiers of Business Research in China, 14(19), 1-25. DOI: $10.1186 / \mathrm{s} 11782-020-00087-1$

Hair, J., Anderson, R., Tatham, R. y Black, W. (2008). Análisis multivariante, 5ta ed., Madrid, España: Prentice Hall.

Hamann, P. M. (2017). Towards a contingency theory of corporate planning: a systematic literature review. Management Review Quarterly, 67, 227-289. DOI: 10.1007/s11301-017-0132-4 
La gestión del cambio, la colaboración virtual y la agilidad estratégica organizacional de empresas mexicanas ante los impactos por el COVID-19

Helfat, C., Finkelstein, S., Mitchell, W., Peteraf, M., Singh, H., Teece, D. y Winter, S. (2007). Dynamic Capabilities: Understanding Strategic Change in Organizations. Malden, MA: Blackwell.

Heller, R. (2010). A cost-benefit analysis of face-to-face and virtual communication: Overcoming the challenges. Ithaca, NY: Cornell University, ILR School, Center for Advanced Human Resource Studies. Disponible en https://digitalcommons.ilr.cornell.edu/cahrswhitepapers/7/

Ivory, S. y Brooks, S. (2018). Managing Corporate Sustainability with a Paradoxical Lens: Lessons from Strategic Agility. Journal of Business Ethics. 148. 1-15. DOI: 10.1007/s10551-0173583-6

Kane, G. C., Palmer, D., Phillips, A. N., Kiron, D., y Buckley, D. (2015). Strategy, not technology, drives digital transformation. MIT Sloan Management Review and Deloitte University Press, 14, 1-25. Disponible en: https://sloanreview.mit.edu/projects/strategy-drivesdigital-transformation/

Kanter, R. M., Stein, B. A., y Jick, T. D. (1992). The challenge of organizational change: How companies experience and leaders guide it. New York: Free Press.

Kline, R. (2005). Principles and practice of Structural Equation Modeling. New York: The Guilford Press.

Kniffin, K., Jayanth N., Frederik A., Antonakis, J., Ashford, S., Bakker, A., Bamberger, P., Bapuji, H., Devasheesh P., Choi, V., Creary, S., Demerouti, E., Flynn, E., Gelfand, M., Greer, L., Johns, G., Selin K., Klein, G., Lee, S. Ozcelik, H., Louise P., Rothbard, N., Cort W., Shaw, J., Sirola, N., Connie R., Whillans, A., Wilmot, M. y Vugt, M. (2020). COVID-19 and the Workplace: Implications, Issues, and Insights for Future Research and Action. American Psychologist (2020). DOI: 10.1037/amp0000716

Konrad, A.M. y Linnehan, F. (1995). Formalized HRM structures: Coordinating equal employment opportunity or concealing organizational practice? Academy of Management Journal, 38 (3), 787-820. DOI: 10.2307/256746

Labarca, N. (2008). Evolución del pensamiento estratégico en la formación de la estrategia empresarial. Opción, 24(55). 47-68. Disponible en: http://ve.scielo.org/scielo.php?pid=S1012-15872008000100004\&script=sci_abstract 
Lawrence, P. R., y Dyer, D. (1983). Renewing American industry: Organizing for efficiency and innovation. New York: Free Press.

Lawrence, P. R., y Lorsch, J. W. (1967). Organization and environment: Managing diferentiation and integration. Boston, MA: Graduate School of Business Administration, Harvard University.

Leana, C.R., y Barry, B. (2000). Stability and change as simultaneous experiences in organizational life. Academy of Management Review, 25,753-759.

Leedy, P. y Ormrod, J. E. (2001). Practical Research. Planning and Design. $7^{\mathrm{a}}$ ed. USA: Merrill Prentice Hall.

Lüscher, L.S. y Lewis, M.W. (2008). Organizational change and managerial sensemaking: working through paradox. Academy of Management Journal, 51(2), 221-240. DOI: 10.5465/AMJ.2008.31767217

Lüscher, L.S. y Lewis, M.W. (2008). Organizational change and managerial sensemaking: working through paradox. Academy of Management Journal, 51 (2), 221-240. https://doi.org/10.5465/amj.2008.31767217

MacKay, R.B. y Chia, R. (2013). Choice, chance, and unintended consequences in strategic change: a process understanding of the rise and fall of NorthCo Automotive. Academy of Management Journal, 56 (1), 208-230. https://doi.org/10.5465/amj.2010.0734

Maguiña, C., Gastelo, R. y Tequen, A. (2020). Revista Médica Herediana, 2020, 31(2), 1-9.

Martín, Q., Cabero, A., De Paz, Y. R. (2008). Tratamiento estadístico de datos con SPSS. Madrid, España: Thomson Learning.

McCann, J. (2004). Organizational effectiveness: Changing concepts for changing environments. Human Resource Planning, 27(1), 42-50. Disponible en: https://www.questia.com/library/p2139/human-resource-planning/i2893063/vol-27-no-1march

McKinley, W., y Scherer, A. (2000). Some unanticipated consequences of organizational restructuring. Academy of Management Review, 25, 735-752. DOI: 10.2307/259202

Mintzberg, J. (1990). El Proceso Estratégico. México: Prentice Hall Hispanoamericana.

Nasim, S. y Sushil (2014). Flexible strategy framework for manging continuity and change en egovernemt. 
La gestión del cambio, la colaboración virtual y la agilidad estratégica organizacional de empresas mexicanas ante los impactos por el COVID-19

https://www.researchgate.net/publication/290463860_Flexible_Strategy_Framework_for_ Managing_Continuity_and_Change_in_E-Government/citation/download

Nunnally J.C. y Bernstein, I.J. (1995). Teoría Psicométrica. México: Mac Graw Hill.

ONU (2020). Noticias. Disponible en: https://news.un.org/es/tags/pandemia/date/2020-06

Organización Panamericana de la Salud (2001). Manual de comunicación social para programas de promoción de la salud de los adolescentes. Washington D.C.: Organización Panamericana de la Salud. Disponible en: http://www.nutricionenmovimiento.org.mx/dif/images/manual_comunicacion_salud_OPS .pdf

Ortlepp, K., y Hloma, X. (2006). People management implications of virtual workplace arrangements. Acta Commercii. 6. DOI: 10.4102/ac.v6i1.86

Pérez, C. (2008). Minería de datos: técnicas y herramientas. Alicante, España: Ediciones Paraninfo. Rogers, D., (2016). The digital transformation playbook: Rethink your business for the digital age. New York: Columbia University Press.

Stoker, J. I., Garretsen, H., y Soudis, D. (2019). Tightening the leash after a threat: A multi-level event study on leadership behavior following the financial crisis. The Leadership Quarterly, 30(2), 199-214. DOI: 10.1016/j.leaqua.2018.08.004

Sturdy, A. y Grey, C. (2003). Beneath and beyond organizational change management: exploring alternatives. Organization, 10, 651-662. DOI: $10.1177 / 13505084030104006$

Shipp, A. J., y Jansen, K. J. (2011). Reinterpreting time in Fit theory. Academy of Management Review, 76-101. https://doi.org/10.5465/AMR.2011.55662565

Sushil. (2005). A Flexible Strategy Framework for Managing Continuity and Change. International Journal of Global Business and Competitiveness, 1(1), 22-32. Disponible en: https://www.researchgate.net/publication/303210963_A_flexible_strategy_framework_for _managing_continuity and_change/citation/download

Teece, D.J., Peteraf M., y Leih, S. (2016). Dynamic Capabilities and organizational agility: risk, uncertainty, and strategy in the innovation economy. California Management Review 58(4),13-35. DOI: 10.1525/cmr.2016.58.4.13

Van Oosterhout M., Waarts, E. y Van Hillegersberg, J. (2006). Change factors requiring agility and implications for IT. European Journal of Information Systems 15(2), 132-145. DOI: 10.1057/palgrave.ejis.3000601 
Vial, G. (2019). Understanding digital transformation: A review and a research agenda. The Journal of Strategic Information Systems, 28(2), 118-144. DOI:10.1016/j.jsis.2019.01.003

Walter, A. (2020). Organizational agility: ill-defined and somewhat confusing? A systematic literature review and conceptualization. Management Review Quarterly. DOI: $\underline{10.1007 / \mathrm{s} 11301-020-00186-6}$

Warner, K. S. R., y Maximilian, W. (2018). Building dynamic capabilities for digital transformation: An ongoing process of strategic renewal. Long Range Planning, 12(52), $326-349$.

Disponible

en: https://www.wcomc.org/sites/default/files/civicrm/persist/contribute/files/20\%20Warner $\% 20$ and $\% 20$ Wager\%20Building\%20dynamic\%20capabilities\%20for\%20digital\%20trans formation\%20_1_.pdf

Weber, Y., y Tarba, S. Y. (2014). Strategic agility: A state of the art. California Management Review, 56(3), 5-12. DOI:10.1525/cmr.2014.56.3.5

Wenzel, M., Stanske, S., y Lieberman, M. B. (2020). Strategic responses to crisis. Strategic Management Journal, 41, 7-18. DOI: 10.1002/smj.3161.

Woods D.D. (2020). The strategic agility gap: How organizations are slow and stale to adapt in turbulent worlds. En: Journé B., Laroche H., Bieder C., Gilbert C. (eds) Human and Organisational Factors. SpringerBriefs in Applied Sciences and Technology. Springer, Cham. DOI:10.1007/978-3-030-25639-5_11

Zeller, D. (2013). The Virtual Workforce: Adapting Human Resource Policies. Disponible en: https://www.researchgate.net/publication/280051176_The_Virtual_Workforce_Adapting Human_Resource_Policies/citation/download 
La gestión del cambio, la colaboración virtual y la agilidad estratégica organizacional de empresas mexicanas ante los impactos por el COVID-19

\section{Anexo}

Reactivos o Ítems

\section{Variable}

\begin{tabular}{|c|c|}
\hline Variable & Reactivo o Ítem \\
\hline \multirow{6}{*}{ Gestión del cambio } & R1. Ante la pandemia, en su empresa se motivó el deseo y la necesidad del cambio \\
\hline & R2. Ante la pandemia, en su empresa se creo un sistema de soporte para los agentes del cambio \\
\hline & $\begin{array}{l}\text { R3. Ante la pandemia, en su empresa para lograr el cambio se construyó una ideología de la } \\
\text { empresa }\end{array}$ \\
\hline & R4. Ante la pandemia, en su empresa para lograr el cambio se planeó el futuro deseado \\
\hline & R5. Ante la pandemia, en su empresa para lograr el cambio se planearon actividades \\
\hline & $\begin{array}{l}\text { R6. Ante la pandemia, en su empresa para lograr el cambio se incidió en el compromiso } \\
\text { personal }\end{array}$ \\
\hline \multirow{4}{*}{ Colaboración virtual } & $\begin{array}{l}\text { R11. Ante la pandemia, para la colaboración virtual en su empresa, coincidían el tipo de } \\
\text { tecnología de la información y de las comunicaciones con la tarea principal a desarrollar }\end{array}$ \\
\hline & $\begin{array}{l}\text { R12. Ante la pandemia, las habilidades de colaboración virtual se desarrollaron a través de una } \\
\text { capacitación formal abarcando las TIC's }\end{array}$ \\
\hline & $\begin{array}{l}\text { R13. Ante la pandemia, la integración y la colaboración en los equipos virtuales permitieron a } \\
\text { los empleados desarrollar una mejor comprensión compartida de sus tareas, objetivos y normas } \\
\text { sociales }\end{array}$ \\
\hline & $\begin{array}{l}\text { R14. Ante la pandemia, la integración y la colaboración en los equipos virtuales permitieron a } \\
\text { los empleados construir lazos sociales más fuertes y una identidad de equipo compartida }\end{array}$ \\
\hline \multirow{4}{*}{ Transformación digital } & $\begin{array}{l}\text { R7. Después de la pandemia, su empresa fortalecerá la aplicación de las tareas de oficina en } \\
\text { línea }\end{array}$ \\
\hline & $\begin{array}{l}\text { R8. Después de la pandemia, su empresa mejorará la digitalización de los canales de la cadena } \\
\text { de suministro }\end{array}$ \\
\hline & $\begin{array}{l}\text { R9. Después de la pandemia, su empresa adoptará plataformas digitales, como plataformas de } \\
\text { comunicación digital }\end{array}$ \\
\hline & $\begin{array}{l}\text { R10. Después de la pandemia, su empresa adoptará infraestructuras digitales, como sistemas } \\
\text { de tecnología digital }\end{array}$ \\
\hline \multirow{4}{*}{$\begin{array}{l}\text { Agilidad estratégica organizacional } \\
\text { ante la pandemia }\end{array}$} & R15. Ante la pandemia, su empresa adoptó el teletrabajo en línea \\
\hline & $\begin{array}{l}\text { R16. Ante la pandemia, su empresa optimizó los modelos de negocio para captar las nuevas } \\
\text { necesidades de los clientes }\end{array}$ \\
\hline & R17. Ante la pandemia, su empresa invirtió en innovación tecnológica \\
\hline & mia, su empresa se diversificó en nuevas áreas de negocios \\
\hline
\end{tabular}

